\title{
Two Theorems on Lattice Expansions
}

\author{
I. Daubechies, Member, IEEE, and A. J.E. M. Janssen, Senior Member, IEEE
}

\begin{abstract}
It is shown that there is a trade-off between the smoothness and decay properties of the dual functions, occurring in the lattice expansion problem. More precisely, it is shown that if $g$ and $\tilde{g}$ are dual, then 1 ) at least one of $H^{1 / 2} g$ and $H^{1 / 2} \tilde{g}$ is not in $\left.L^{2}(\mathbb{R}), 2\right)$ at least one of $H g$ and $\tilde{g}$ is not in $L^{2}(\mathbb{R})$. Here, $H$ is the operator $-1 /\left(4 \pi^{2}\right) d^{2} /\left(d t^{2}\right)+t^{2}$. The first result is a generalization of a theorem first stated by Balian and independently by Low, which was recently rigorously proved by Coifman and Semmes; a new, much shorter proof was very recently given by Battle. Battle suggests a theorem of type (i), but our result is stronger in the sense that certain implicit assumptions made by Battle are removed. Result 2) is new and relies heavily on the fact that, when $G \in W^{2,2}(S)$ with $S=\left[-\frac{1}{2}, \frac{1}{2}\right] \times\left[-\frac{1}{2}, \frac{1}{2}\right]$ and $G(0)=0$, then $1 / G \notin L^{2}(S)$. The latter result was not known to us and may be of independent interest.
\end{abstract}

Index Terms - Gabor transformation, frame, orthonormal basics, and time-frequency localization.

\section{INTRODUCTION}

W E CONSIDER in this note expansions of the type

$$
f(t) \sim \sum_{n, m} c_{n m} g_{n m}(t), \quad t \in \mathbb{R}
$$

where $f \in L^{2}(\mathbb{R})$,

$$
g_{n m}(t)=e^{-2 \pi i m \omega t} g(t+n), \quad t \in \mathbb{R}, n, m \in \mathbb{Z},
$$

$g \in L^{2}(\mathbb{R})$ is a fixed function of time, usually well concentrated in time and frequency, and $\omega$ is a fixed real number $\neq 0$. For $\omega<1$, many choices for $g$ lead to convergent expansions for all $f \in L^{2}(\mathbb{R})$, [8]. In this paper, we restrict ourselves to the case $\omega=1$, corresponding to lattices with the largest possible mesh size.

In (1.1), the coefficients $c_{n m}$ depend on both $f$ and $g$. Problems related to the present one were considered (for the case of Gaussian $g$ ) by Von Neumann in a quantum mechanical context [16], by Gabor in the context of efficient data transmission [9], by Perelomov [17], Bargmann, Butera, Girardello, and Klauder [3], and by Bacry, Grossmann, and Zak [1], who all gave completeness properties of the set of $g_{n m}$ 's. The problem of determining the coefficients $c_{n m}$ in the expansion (1.1) became tractable notably through the work of Zak on solid-state physics related problems [1], [20], [21], of Bastiaans on optical signal description [4], [5], and of Janssen who gave rigorous proofs of existence and

Manuscript received September 13, 1988.

1. Daubechies is with AT\&T Bell Laboratories, 600 Mountain Avenue, Rm. 2C-371, Murray Hill, NJ 07974-2070.

A. J.E. M. Janssen is with Philips Research Laboratories, 5600 JA Eindhoven, The Netherlands.

IEEE Log Number 9203010 convergence of the expansions $(1.1)$ in $L^{2}(\mathbb{R})$ and other spaces of (generalized) functions [11]-[13]. In this context, Daubechies and Grossmann [7] exploited the notion of frame that indicates a set of functions $g_{n m}$ as in (1.2) such that for some $A>0, B>0$,

$$
A\|f\|^{2} \leqq \sum_{n, m}\left|\left(f, g_{n m}\right)\right|^{2} \leqq B\|f\|^{2},
$$

for all $f \in L^{2}(\mathbb{R})$. $H \cdot\|\cdot\|$ and $(\cdot, \cdot)$ denote ordinary norm and inner produs $\quad L^{2}(\mathbb{R})$. It is amply demonstrated in the comprehensive $\quad[8]$ that for numerically reliable expansion of $f$ as in (. e needs to consider $g$ 's such that the set of $g_{n m}$ 's const1. a frame. An even more desirable case occurs when the cot stants $A$ and $B$ are equal. Then the $g_{n m}$ 's are said to constitute a tight frame, and the $g_{n m}$ 's are orthogonal.

The procedure of finding the coefficients $c_{n m}$ in (1.1) is as follows. Consider the ma- ing $Z$ defined for $f \in L^{2}(\mathbb{R})$ by

$$
(Z f)(\tau, \Omega)=\sum_{k=-\infty}^{\infty}(\tau+k) e^{-2 \pi i k \Omega}, \quad \tau, \Omega \in \mathbb{R} .
$$

This mapping has several names, such as Gel'fand mapping [10], [19], Weil-Brézin mapping [18], Zak transform [13], [14], while it seems that Gauss was already aware of some of its properties [18]. We shall call $Z$ the Zak transform, since Zak seems to be the first one to exploit the transform systematically in the context of completeness and expansion problems. For a survey of the numerous properties of the Zak transform we refer to [14]. The relevant property of $Z$. for the expansion problem is that

$$
\left(Z g_{n m}\right)(\tau, \Omega)=e^{-2 \pi i n+\pi i m \Omega}(Z g)(\tau, \Omega) .
$$

Hence, we have, at least formally,

$$
c_{n m}=\iint e^{2 \pi i m \tau-2 \pi i n \Omega} \frac{(Z f)(\tau, \Omega)}{(Z g)(\tau, \Omega)} d \tau d \Omega .
$$

In (1.6), the integratior: $\because$ over any unit square $(Z f / Z g$ is periodic with period 1, , th its variables). To introduce the notion of dual function w note the property that $Z$ is a Hilbert space isomorphism betwes $L^{2}(\mathbb{R})$ and the set of all functions $F(\tau, \Omega)$ such that

$$
F(\tau, \Omega+1)=F(\tau, \Omega), \quad F(\tau+1, \Omega)=e^{2 \pi i \Omega} F(\tau, \Omega) .
$$

In the latter set of functions the inner product of an $F$ and $G$ satisfying the (quasi-) per: licity relations in (1.7) is given by

$$
(F, G)=\iint F(\tau, \Omega) G^{*}(\tau, \Omega) d \tau d \Omega,
$$


where the integral is over any unit square and the asterisk denotes complex conjugation. In particular, for any $f_{1}, f_{2} \in$ $L^{2}(\mathbb{R})$, we have

$$
\left(f_{1}, f_{2}\right)=\left(Z f_{1}, Z f_{2}\right) .
$$

Now, the function $1 /(Z g)^{*}$ satisfies the relations (1.7), and, if it is square integrable over a unit square, there is a unique $\dot{\tilde{g}} \in L^{2}(\mathbb{R})$ such that

$$
Z \tilde{g}=\frac{1}{(Z g)^{*}} .
$$

This $\tilde{g}$ is called the dual function, and $\tilde{g}_{n m}$ constitute the dual frame. We observe that $\tilde{\tilde{g}}=g$, and that

$$
\left(g, \tilde{g}_{n m}\right)=\delta_{n 0} \delta_{m 0}=\left(\tilde{g}, g_{n m}\right),
$$

with $\delta$ the Kronecker function.

It follows from (1.5), (1.6), and (1.10) that the coefficients $c_{n m}$ can be expressed as

$$
c_{n m}=\left(f, \tilde{g}_{n m}\right) .
$$

Furthermore, the conditions of being a frame and a tight frame can be expressed in terms of the Zak transform as

$$
\operatorname{ess} \sup |Z g|<\infty, \quad \operatorname{ess} \sup |Z g|>0
$$

and

$$
\text { ess } \sup |Z g|=\operatorname{essinf}|Z g|<\infty,
$$

respectively, (see [8]).

The two main theorems of this paper read as follows. With the notation, $H=-\frac{1}{4 \pi^{2}} \frac{d^{2}}{d t^{2}}+t^{2}$ for the Hermite operator, we have the following.

Theorem I: If $g$ and $\tilde{g}$ are dual functions in the sense previously explained, then $H^{1 / 2} g$ and $H^{1 / 2} \tilde{g}$ cannot both be in $L^{2}(\mathbb{R})$.

Theorem II: Under the same assumptions, $H g$ and $\tilde{g}$ cannot both be in $L^{2}(\mathbb{R})$.

Hence, in a sense, $g$ and $\tilde{g}$ cannot both be smooth and rapidly decaying. That such results can be expected is seen as follows. Assume that $g$ is such that $Z g$ is continuous; this holds when $g$ is continuous and decays sufficiently rapidly, e.g., like $1 /(1+|t|)^{\alpha}$ with $\alpha>1$. It is a curious property of the Zak transform that then $Z g$ has at least one zero in the unit square [1], [13]. Hence, ess $\sup |Z \tilde{g}|=\infty$. And when $Z g$ is continuously differentiable, we even have that $1 / Z g$ is not square integrable over the unit square.

Note that nevertheless Theorems I and II are nontrivial since $H^{1 / 2} g \in L^{2}(\mathbb{R})$ does not imply that $Z g$ is continuous, and $H g \in L^{2}(\mathbb{R})$ does not imply that $Z g$ is continuously differentiable.

Recently, some results like ours have been proved. Balian [2] and Low [15] both argued that at least one of $t g(t)$ and $g^{\prime}(t)$ is not in $L^{2}(\mathbb{R})$ when $g_{n m}$ constitutes a tight frame. Their argument was made rigorous and extended by Coifman and Semmes to include the case of nontight frames; this is presented by Daubechies in [8]. Finally, an independent, more elegant, proof of the Balian-Low result was given by Battle in [6].

To see what the novelty of the present paper is, we give some further preliminary remarks. The conditions

a) $H^{1 / 2} g \in L^{2}(\mathbb{R})$,

b) $t g(t) \in L^{2}(\mathbb{R}), \quad g^{\prime}(t) \in L^{2}(\mathbb{R})$,

c) $\frac{\partial Z g}{\partial \tau} \in L^{2}(S), \frac{\partial Z g}{\partial \tau} \in L^{2}(S)$, with $S$ any unit square (i.e., $Z G \in W^{2,1}(S)$ ),

are equivalent.

That a) and b) are equivalent is a standard fact; that b) and c) are equivalent follows from (2.1) and (2.9). Similarly, the conditions

e) $H g \in L^{2}(\mathbb{R})$

f) $t^{2} g(t) \in L^{2}(\mathbb{R}), \quad g^{\prime \prime}(t) \in L^{2}(\mathbb{R})$,

g) $\frac{\partial^{2} Z_{g}}{\partial \tau^{2}} \in L^{2}(S), \quad \frac{\partial^{2} Z_{g}}{\partial \Omega^{2}} \in l^{2}(S), \quad \frac{\partial^{2} Z g}{\partial \tau \partial \Omega} \in L^{2}(S)$ (i.e., $Z g \in W^{2,2}(S)$ ),

are equivalent. Now, when $g_{n m}$ constitutes a frame and $Z g \in$ $W^{2,1}(S)$, it follows from $\operatorname{essinf}|Z g|>0$ and

$$
\begin{aligned}
& \frac{\partial}{\partial \tau}\left(\frac{1}{Z g}\right)=-\left(\frac{1}{Z g}\right)^{2} \frac{\partial Z g}{\partial \tau}, \\
& \frac{\partial}{\partial \Omega}\left(\frac{1}{Z g}\right)=-\left(\frac{1}{Z g}\right)^{2} \frac{\partial Z g}{\partial \Omega}
\end{aligned}
$$

that

$$
\frac{1}{(Z g)^{*}}=Z \tilde{g} \in W^{2,1}(S) .
$$

That is, Theorem I implies the Coifman-Semmes result, and, a fortiori, the Balian-Low-Battle result. The Theorem II is entirely new as far as we know. While our proof of Theorem I uses a little trick that can be found in Battle's paper, the proof of Theorem II is based on the two facts that

a) when $Z g \in W^{2,2}(S)$ then $Z g$ is continuous and has a zero in $S$,

b) when $G \in W^{2,2}(S)$ has a zero then $1 / G \notin L^{2}(S)$.

We were unable to find the result (b) in the literature, and it may be of some independent interest.

Theorems I and II may be viewed as no-go theorems, excluding the possibility of numerically stable expansions of type (1.1) with respect to $g_{n m}$ in (1.2) with $\omega=1$, which are well-localized in both time and frequency. This can be avoided by using expansions with tighter lattices, corresponding to the choice $\omega<1$, [8]. It is well known that the dual function $\tilde{g}$ has many singular features [4], [5] if $g$ is Gaussian. Our Theorem II generalizes the result in [13] that $\tilde{g}$ is not square integrable.

\section{ProOF OF THEOREM I}

As explained in Section I, we must take a $g \in L^{2}(\mathbb{R})$ with $Z g, Z \tilde{g} \in W^{2,1}(S)$ and show that this leads to a contradiction. Denote

$$
(Q g)(t)=t g(t), \quad(P g)(t)=\frac{1}{2 \pi i} g^{\prime}(t), \quad \text { etc. }
$$

As in Battle's proof, we shall show that

$$
(Q g, P \tilde{g})=(P g, Q \tilde{g})
$$


- (by assumption, all four functions involved in (2.2) are in $\left.L^{2}(\mathbb{R})\right)$. This implies that $(g, \tilde{g})=0$, which is absurd by (1.11).

To demonstrate (2.2), we need the auxiliary results

$$
\begin{aligned}
& (Q g, P \tilde{g})=\sum_{n, m}\left(Q g, \tilde{g}_{n m}\right)\left(g_{n m}, P \tilde{g}\right), \\
& (P g, Q \tilde{g})=\sum_{n, m}\left(P g, \tilde{g}_{n m}\right)\left(g_{n m}, Q \tilde{g}\right) .
\end{aligned}
$$

In [6], the validity of the expansions (2.3), (2.4) was implicitly assumed (and not proved as is done here).

The relation (2.3) follows from the fact, to be proved below, that $Z Q g / Z g, Z P \tilde{g} / Z \tilde{g} \in L^{2}(S)$. Indeed, it then follows from $Z g \cdot(Z \tilde{g})^{*}=1$ that

$$
(Q g, P \tilde{g})=(Z Q g, Z P \tilde{g})=(Z Q g / Z g, Z P \tilde{g} / Z \tilde{g}) .
$$

The right-hand side of (2.5) equals the right-hand side of (2.3), since $\left(Q g, \tilde{g}_{n m}\right)$ and $\left(P \tilde{g}, g_{n m}\right)$ are the Fourier coefficients of $Z Q g / Z g$ and $Z P \tilde{g} / Z \tilde{g}$ by (1.6) and (1.12). Similarly, $Z P g / Z g, Z Q \tilde{g} / Z \tilde{g} \in L^{2}(S)$ implies that (2.4) holds. have

We shall show now that $Z Q g / Z g, Z P \tilde{g} / Z \tilde{g} \in L^{2}(S)$. We

$$
\left(\frac{1}{Z g}\right)^{2} \frac{\partial Z g}{\partial \Omega}=-\frac{\partial}{\partial \Omega}\left(\frac{1}{Z g}\right)=-\frac{\partial}{\partial \Omega}(Z \tilde{g})^{*} \in L^{2}(S) .
$$

It follows from the Cauchy-Schwarz inequality that

$$
\left|\frac{1}{Z g} \frac{\partial Z g}{\partial \Omega}\right|=\left|\left(\frac{1}{Z g}\right)^{2} \frac{\partial Z g}{\partial \Omega}\right|^{1 / 2}\left|\frac{\partial Z g}{\partial \Omega}\right|^{1 / 2} \in L^{2}(S),
$$

and, similarly,

$$
\left|\frac{1}{Z \tilde{g}} \cdot \frac{\partial Z \tilde{g}}{\partial \tau}\right| \in L^{2}(S)
$$

Since

$$
Z Q g=\frac{1}{2 \pi i} \frac{\partial Z g}{\partial \Omega}+\tau(Z g)(\tau, \Omega), \quad Z P \tilde{g}=\frac{1}{2 \pi i} \frac{\partial Z \tilde{g}}{\partial \tau}
$$

it follows that $Z Q g / Z g, Z P \tilde{g} / Z \tilde{g} \in L^{2}(S)$, as claimed.

To show (2.2), it suffices to prove that

$$
\begin{aligned}
& \left(Q g, \tilde{g}_{n m}\right)=\left(g_{-n,-m}, Q \tilde{g}\right), \\
& \left(P g, \tilde{g}_{n m}\right)=\left(g_{-n,-m}, P \tilde{g}\right) .
\end{aligned}
$$

We have

$$
\begin{aligned}
\left(Q g, \tilde{g}_{n m}\right) & =\int t g(t)\left(e^{-2 \pi i m t} \tilde{g}(t+n)\right)^{*} d t \\
& =\int(t-n) g(t-n) e^{2 \pi i m t} \tilde{g}^{*}(t) d t \\
& =\left(g_{-n,-m}, Q \tilde{g}\right)-n\left(g_{-n,-m}, \tilde{g}\right) .
\end{aligned}
$$

Together with (1.11), this implies the first part of (2.10). The second part of (2.10) follows from the first part by noting that, with $\mathcal{F}$ the Fourier transform,

$$
\mathcal{F} P=Q \mathcal{F}, \quad \mathcal{F} g_{n m}=(\mathcal{F} g)_{-m,-n},
$$

and the fact that $\mathcal{F} g$ and $\mathcal{F} \tilde{g}$ are dual. This establishes (2.2).

We conclude the proof of Theorem I by showing that $(2.2)$ implies that $(g, \tilde{g})=0$. We have by assumption

$$
t \frac{d}{d t}\left(g(t) \tilde{g}^{*}(t)\right)=\operatorname{tg}(t)\left(\tilde{g}^{\prime}(t)\right)^{*}+t g^{\prime}(t) \tilde{g}^{*}(t) \in L^{1}(\mathbb{R}) .
$$

The right-hand side function in (2.13) equals

$$
-2 \pi i\left(Q g \cdot(P \tilde{g})^{*}-P g \cdot(Q \tilde{g})^{*}\right) .
$$

We now have, for all $a<b$

$$
\int_{a}^{b} t \frac{d}{d t}\left(g(t) \tilde{g}^{*}(t)\right) d t=\left.t g(t) \tilde{g}^{*}(t)\right|_{a} ^{b}-\int_{a}^{b} g(t) \tilde{g}^{*}(t) d t .
$$

When $a \rightarrow-\infty, b \rightarrow \infty$, the left-hand side of (2.15) tends to 0 by (2.2), (2.13) and (2.14), and the integral on the right-hand side tends to $(g, \tilde{g})$. Hence,

$$
\left.\lim _{b \rightarrow \infty, a \rightarrow-\infty} t g(t) \tilde{g}^{*}(t)\right|_{a} ^{b}
$$

exists as well and equals 0 since $t g(t) \tilde{g}^{*}(t) \in L^{1}(\mathbb{R})$. Therefore, $(g, \tilde{g})=0$, and the proof of Theorem I is complete.

\section{Proof of Theorem II}

As already explained at the end of Section I, it is sufficient to show the following result.

Proposition: Assume $G \in W^{2,2}(S)$, where $S=\left[-\frac{1}{2}, \frac{1}{2}\right] \times$ $\left[-\frac{1}{2}, \frac{1}{2}\right]$, and $G(0,0)=0$. Then, $1 / G \notin L^{2}(S)$.

Proof: For notational convenience we write $x=(\tau, \Omega) \in$ $\mathbb{R}^{2}$, and we denote by $|\cdot|$ and Euclidean norm and inner product in $\mathbb{R}^{2}$, respectively. Let $0<r<\frac{1}{2}$. We (re)define $G(x)$ for $|x| \geqq r$ such that the resulting function, again denoted by $G$, is in $W^{2,2}\left(\mathbb{R}^{2}\right)$. When $\hat{G}(\xi), \xi \in \mathbb{R}^{2}$ is the Fourier transform if $G$, we have

$$
\iint\left(1+|\xi|^{2}\right)^{2}|\hat{G}(\xi)|^{2} d \xi<\infty
$$

Now, by Fourier inversion,

$$
G(x)=G(x)-G(0)=\iint\left(e^{2 \pi i x \cdot \xi}-1\right) \hat{G}(\xi) d \xi .
$$

Hence, by the Cauchy-Schwarz inequality,

$$
\begin{aligned}
|G(x)|^{2} \leqq & \iint \frac{\left|e^{2 \pi i x \cdot \xi}-1\right|^{2}}{\left(1+|\xi|^{2}\right)^{2}} d \xi \\
& \cdot \iint\left(1+|\xi|^{2}\right)^{2}|\hat{G}(\xi)|^{2} d \xi .
\end{aligned}
$$

We have for the first integral $I_{1}$ in (3.3)

$$
I_{1}=4 \iint \frac{\sin ^{2} \pi x \cdot \xi}{\left(1+|\xi|^{2}\right)^{2}} d \xi=4 \iiint_{|\xi| \leqq \frac{1}{|x|}}+4 \iint_{|\xi| \geqq \frac{1}{|x|}} .
$$

The first integral $I_{2}$ in (3.4) satisfies

$I_{2} \leqq \pi^{2}|x|^{2} \quad \iint_{|\xi| \leqq \frac{1}{|x|}} \frac{|\xi|^{2}}{\left(1+|\xi|^{2}\right)^{2}} d \xi \leqq \pi^{3}|x|^{2} \log \left(1+\frac{1}{|x|^{2}}\right)$. 
The second integral $I_{3}$ in (3.4) satisfies

$$
I_{3} \leqq \iint_{|\xi| \geqq \frac{1}{|x|}} \frac{d \xi}{\left(1+|\xi|^{2}\right)^{2}}=\frac{\pi|x|^{2}}{1+|x|^{2}}
$$

Hence, by (3.1), (3.3), (3.5), and (3.6).

$$
|G(x)|^{2}=0\left(|x|^{2} \log \frac{1}{|x|^{2}}\right), \quad|x| \leqq \frac{1}{2} .
$$

Since

$$
\iint_{|x| \leqq \frac{1}{2}} \frac{d x}{|x|^{2} \log \frac{1}{|x|^{2}}}=\infty
$$

the proposition follows.

\section{ACKNOWLEDGMENT}

The authors wish to thank Ph. Tchamitchian (I.D.) and J. de Graaf and S.J. L. van Eijndhoven (A.J.E.M.J.) for stimulating discussions on the two theorems.

\section{REFERENCES}

[1] H. Bacry, A. Grossmann, and J. Zak, "Proof of completeness of lattice states in the $k q$-representation," Phys. Rev., Ser. B, vol. 3, no. 12, pp. $1118-1120,1975$.

[2] R. Balian, "Un principe d'incertitude fort en théorie du signal ou en mécanique quantique," C.R. Acad. Sc. Paris, 292, série 2, 1357-1362, 1987.

[3] V. Bargmann, P. Butera, L. Girardello, and J. R. Klauder, "On the completeness of the coherent states," Rep. Math. Phys., vol. 2, pp. 221-228, 1971.
[4] M. J. Bastiaans, "Gabor's signal expansion and degrees of freedom of a . signal," Optica Acta, vol. 29, 1223-1229, 1982.

[5] _ "Optical generation of Gabor's expansion coefficients for rastered signals," Optica Acta, vol. 29, pp. 1349-1357, 1982.

[6] G. Battle, "Heisenberg proof of Low's theorem," Lett. Math. Phys., vol. 15 , pp. $175-179,1988$.

[7] I. Daubechies and A. Grossman, "Frames of entire functions in the Bargmann space," Commun. Pure Appl. Math., vol. 41, pp. 151-164, 1988.

[8] I. Daubechies, "The wavelet transform, time-frequent localization, and signal analysis," IEEE Trans. Inform. Theory, vol. 36, pp. 961-1005, Sept. 1990 .

[9] D. Gabor, "Theory of communication," J. Inst. Elect. Eng. (London), vol. 93, no. III, pp. 429-457, 1946.

[10] I. M. Gel'fand, "Eigenfunction expansions for an equation with periodic coefficients," Dokl. Akad. Nauk SSSR, vol. 76, pp. 1117-1120, 1950 (in Russian).

[11] A.J.E.M. Janssen, "Weighted Wigner distributions vanishing on lattices," J. Math. Anal. Appl., vol. 80, pp. 156-167, 1981.

[12] "Gabor representation of generalized functions," J. Math. Anal Appl., vol. 83, pp. 377-394, 1981.

[13] - "Bargmann transform, Zak transform, and coherent states," $J$ Math. Phys., vol. 23, pp. 720-731, 1982

[14] "The Zak transform: A signal transform for sampled timecontinuous signals," to appear in Philips J. Res., vol. 43, no. 1, 1988.

[15] F. Low, "Complete sets of wave-packets," in A Passion for PhysicsEssays in Honor of Geoffrey Chew. Singapore: World Scientific, 1985, pp. $17-22$.

[16] J. von Neumann, Mathematical Foundations of Quantum Mechanics. Princeton, NJ: Princeton Univ. Press, 1955, pp. 405-407.

[17] A. M. Perelomov, "On the completeness of a system of coherent states," Theoret. and Math. Phys., vol. 6, pp. 156-164, 1971.

[18] W. Schempp, "Radar ambiguity functions, the Heisenberg group, and holomorphic theta series," Proc. Am. Math. Soc, vol 92, vol. 108, 1984.

[19] M.M. Skriganov, "Geometric and arithmetic methods in the spectral theory of multidimensional periodic operators," Proc. Steklov Inst. Math., vol. 17, Am. Math. Soc., Providence, p. 171, 1987.

[20] J. Zak, "Finite translations in solid-state physics," Phys. Rev. Lett., vol. 19, pp. $1385-1387,1967$.

[21] -Lattice operators in crystals for Bravais and reciprocal vectors," Phys. Rev. B, vol. 12, pp. 3023-3025, 1975 